EPJ Web of Conferences 66, 02089 (2014)

DOI: 10.1051/epjconf/20146602089

(C) Owned by the authors, published by EDP Sciences, 2014

\title{
Exotic decay of hot rotating nuclei near proton drip line
}

J. Ray ${ }^{1}$, U. Datta Pramanik, a, R. K. Bhowmik ${ }^{2}$, I. Ray ${ }^{1}$, A. Rahaman ${ }^{1}$, A. Chakraborty ${ }^{3}$, S. Chakraborty $^{1}$, R. Garg ${ }^{4}$, S. Goyall ${ }^{4}$, S. Ganguly ${ }^{5}$, S. Kumar ${ }^{6}$, S. Mandal ${ }^{4}$, B. Mukherjee ${ }^{3}$, P. Mukherjee $^{7}$, S. Muralithar ${ }^{2}$, D. Negi ${ }^{2}$, M. Saxena ${ }^{4}$, K. Selvakumar ${ }^{6}$, P. Singh ${ }^{6}$, A. K. Singh ${ }^{6}$, and R. P. Singh ${ }^{2}$

\author{
${ }^{1}$ Saha Institute of Nuclear Physics, Kolkata, India \\ ${ }^{2}$ Inter University Accelerator Centre (IUAC), New Delhi, India \\ ${ }^{3}$ Viswa Bharati University, Santiniketan, India \\ ${ }^{4}$ University of New Delhi, New Delhi, India \\ ${ }^{5}$ Chandannagar College, Chandannagar, India \\ ${ }^{6}$ Indian Institute of Technology, Kharagpur, India and \\ ${ }^{7}$ St. Xavier's College, Kolkata, India
}

\begin{abstract}
Hot and rotating exotic ${ }^{124} \mathrm{Ce}$ nucleus near proton drip line has been populated through fusion evaporation reaction of ${ }^{32} \mathrm{~S}$ and ${ }^{92} \mathrm{Mo}$. This exotic nucleus was de-excited by evaporating $\mathrm{p}, \mathrm{n}, \alpha$ and/or light nuclei etc and several exotic nuclei have been populated. The experimentally obtained relative population of those exotic nuclei have been compared with the statistical model calculation. Agreement between experimental and statistical model calculation have been observed for most of the evaporation channels. Huge enhancement in comparison to statistical model calculation have been observed for a few channels related to multiple proton evaporation which could not be explained by using default and modified input parameters in statistical calculation.
\end{abstract}

\section{Introduction}

Decay of hot and rotating compound nucleus far from stability or near drip line is one of the interesting topic in recent years. The de-excitation of compound nucleus, near stability, produced in fusion-evaporation reaction in general follows the standard statistical process. This standard statistical calculation has been implemented in various statistical codes like HIVAP, PACE , ALICE , CASCADE, GEMINI etc. Difference in these codes depend upon some significant ingredients like particle transmission coefficients, level densities, fission barriers etc. which are extracted from different phenomenological models. Radioactive beams are the most suitable to reach the neutron drip line whereas using stable beams proton dripline could be reached through the process of fusionevaporation reaction. Further through fusion evaporation reaction one can populate exotic nuclei near proton drip line with high excitation and large angular momentum. Although fusion-evaporation is a well known and commonly used method to explore stable and neutron deficient nuclei in various mass region, but the de-excitation process of the compound nucleus $(\mathrm{CN})$ in different mass region are

${ }^{\mathrm{a} C}$ Corresponding author, e-mail: ushasi.dattapramanik@saha.ac.in 
yet to be investigated. Although these models predict decay of compound nucleus near stability very well, but near the nuclear drip line the compound nuclear systems are not rigorously studied. Non statistical decay along with the statistical decay in various mass region have been reported earlier[1]. So it would be interesting to check whether the standard statistical model calculations are applicable to the evaporation of the compound nuclei far away from the stability or close to the drip line.

The aim of the present work is to study the de excitation of the highly excited exotic nucleus ${ }^{124} \mathrm{Ce}\left(\mathrm{S}_{n}=12.7 \mathrm{MeV}\right.$ and $\left.\mathrm{S}_{p}=3.4 \mathrm{MeV}\right)$, produced by fusion-evaporation reaction of ${ }^{32} \mathrm{~S}$ and ${ }^{92} \mathrm{Mo}$. The experimental result has been compared with statistical model predictions. The details of the experimental procedure is given in sec 2 . The method of analysis and observations are described in $\sec 3$. The tentative explanation is summarized in $\sec 4$.

\section{Experimental Details}

The experiment was carried out using ${ }^{32} \mathrm{~S}$ beam of energy $150 \mathrm{MeV}$ (from 15 UD Pelletron accelerator) on self - supporting ${ }^{92}$ Mo target of thickness $\sim 7.3 \mathrm{mg} / \mathrm{cm}^{2}$, at the Inter University Accelerator Centre, New Delhi. The beam energy was $\sim 30 \%$ above the Coulomb barrier $(\sim 115 \mathrm{MeV}$ in laboratory frame) and consequently the compound nucleus ${ }^{124} \mathrm{Ce}$ was formed with excitation energy $\sim 63$ $\mathrm{MeV}$ and angular momentum upto $\sim 59 \hbar$. This hot-rotating exotic nucleus was de-excited by evaporation of proton, neutron, alpha and/or light nuclei. As a result a number of exotic nuclei such as ${ }^{121} \mathrm{La},{ }^{120-122} \mathrm{Ba},{ }^{118-121} \mathrm{Cs},{ }^{117-120} \mathrm{Xe},{ }^{115,117} \mathrm{I},{ }^{114} \mathrm{Te}$ etc have been populated in high excited states. These nuclei were de-excited by emitting initially statistical and then later discrete $\gamma$-rays. These deexciting discrete $\gamma$-rays were detected both in singles and $\gamma$ - $\gamma$ coincidence mode by Indian National Gamma Array (INGA)[2], at IUAC New Delhi. The experimental setup with INGA which consists of 12 Compton-suppressed clover detectors, has been shown in fig.1(a). The energy calibration and efficiency measurement of the clover detectors were performed using the ${ }^{133} \mathrm{Ba}$ and ${ }^{152} \mathrm{Eu}$ source of known strength. For data acquisition the electronics comprising of the IUAC Clover module and software CANDLE were used. For analysis of data INGASORT software has been used.

\section{Data analysis and Result}

High spin states of the neutron deficient very exotic nuclei like ${ }^{120} \mathrm{Ba},{ }^{121} \mathrm{Ba},{ }^{122} \mathrm{Ba},{ }^{121} \mathrm{La},{ }^{118} \mathrm{Cs}$, ${ }^{119} \mathrm{Cs},{ }^{120} \mathrm{Cs},{ }^{121} \mathrm{Cs},{ }^{117} \mathrm{Xe},{ }^{118} \mathrm{Xe},{ }^{119} \mathrm{Xe},{ }^{120} \mathrm{Xe},{ }^{115} \mathrm{I},{ }^{117} \mathrm{I},{ }^{114} \mathrm{Te}$ etc. have been populated through evaporation of protons, neutrons, alpha, combination of proton, neutron and alpha, lighter nuclei from the compound nucleus ${ }^{124} \mathrm{Ce}$. All the populated isotopes have been identified by their characteristic $\gamma$-rays and relative population of the isotopes have been measured. Fig. 1(b) shows the isotopes populated in the present experiment.

The population of the isotopes have been measured by intensities of the characteristic $\gamma$-rays of the isotopes. Contamination of the characteristic gamma-ray from other isotopes has been carefully checked with the help of $\gamma-\gamma$ coincidence gated spectra. The population of all the isotopes obtained in this way have been then normalised with respect to the population of ${ }^{120} \mathrm{Cs}\left({ }^{120} \mathrm{Cs}\right.$ should have been populated only due to evaporation of $3 \mathrm{p} 1 \mathrm{n}$ from the compound nucleus). The experimentally obtained relative population of various isotopes have been then compared with the standard statistical model calculation (PACE). For most of the populated nuclei, experimentally obtained relative population are in agreement with the standard statistical model calculation. On the contrary a few isotopes ${ }^{122} \mathrm{Ba}$, ${ }^{121} \mathrm{Cs},{ }^{120} \mathrm{Xe}$ and ${ }^{118} \mathrm{Xe}$ have population hugely enhanced (2 to 3 times) compared to the theoretically predicted population. This interesting behaviour in population of the isotopes have been consistently observed for the experiments with beam energies $135 \mathrm{MeV}, 140 \mathrm{MeV}$ and $145 \mathrm{MeV}$ performed with 


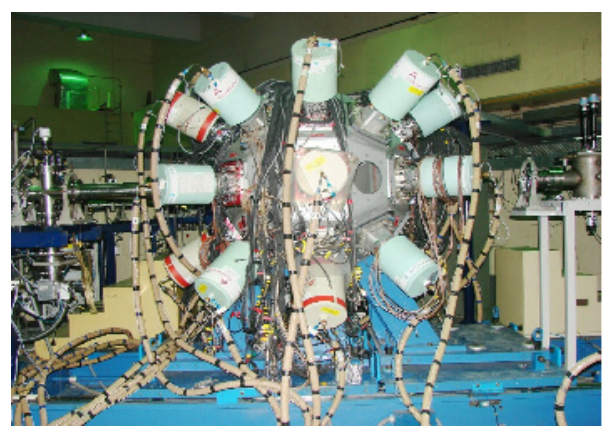

(a)

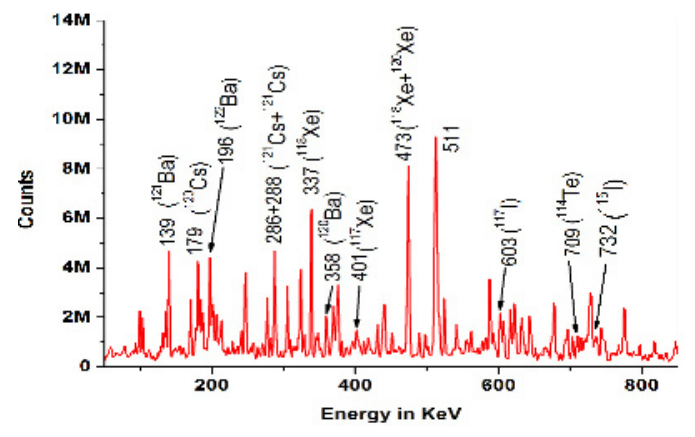

(b)

Figure 1. (a) Experimental setup at IUAC, New Delhi; (b) all-gated spectrum obtained from clover detectors of INGA, showing the isotopes populated in the ${ }^{32} \mathrm{~S}+{ }^{92}$ Mo reaction.

the same target projectile combination, but with thin $\left(\sim 200 \mu \mathrm{g} / \mathrm{cm}^{2}\right)$ target.

Statistical model calculation (PACE) (solid squares) and experimentally obtained (solid circles) rel-

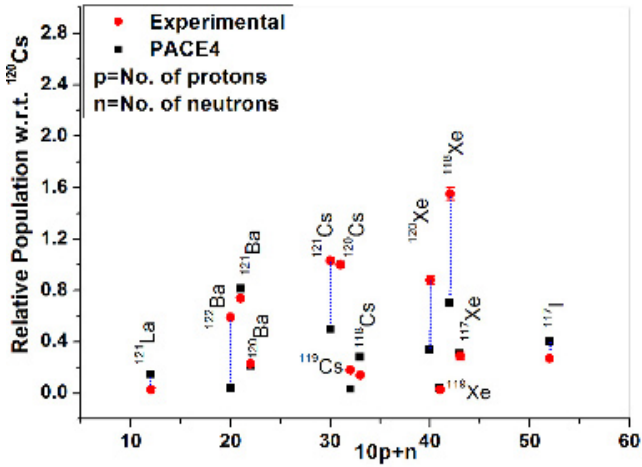

(a)

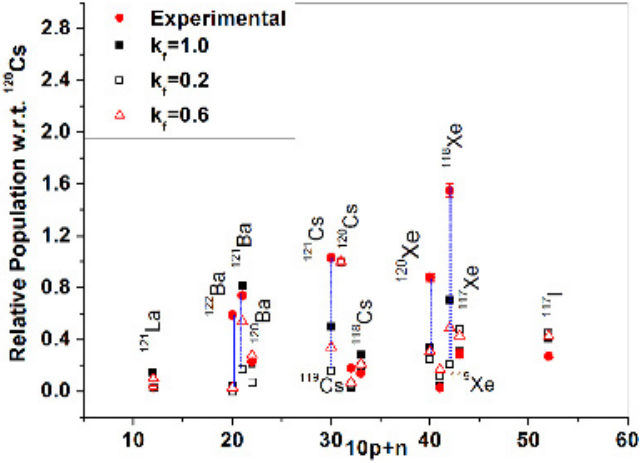

(b)

Figure 2. (a)Statistical model calculation (solid squares) and Experimental (solid circles) relative population (with respect to ${ }^{120} \mathrm{Cs}$ ) of various evaporation residues showing enhanced population of ${ }^{122} \mathrm{Ba},{ }^{121} \mathrm{Cs},{ }^{120} \mathrm{Xe}$ and ${ }^{118} \mathrm{Xe}$; (b)PACE calculation with fission barrier scaling factor $\mathrm{k}_{f}=0.2$ (squares), 0.6 (triangles) and 1.0 (solid squares) compared with present experimental(solid circles) relative population.

ative populations have been shown in fig. 2(a), where the $\mathrm{X}$-axis represents number of protons and neutrons evaporated from the compound nucleus ( $\mathrm{p}=$ no. of evaporated protons, $\mathrm{n}=$ no. of evaporated neutrons) and Y-axis represents the relative population of the isotopes. The comparison between the theoretical and experimental result shows [fig. 2(a)] that the population of most of the nuclei like ${ }^{121} \mathrm{La}(\mathrm{p} 2 \mathrm{n}),{ }^{120} \mathrm{Ba}(2 \mathrm{p} 2 \mathrm{n}$ or $\alpha),{ }^{117} \mathrm{Xe}(4 \mathrm{p} 3 \mathrm{n}),{ }^{117} \mathrm{I}(5 \mathrm{p} 2 \mathrm{n}),{ }^{114} \mathrm{Te}(6 \mathrm{p} 4 \mathrm{n})$ etc. are in agreement with the statistical model calculation whereas unusual enhancement have been observed for ${ }^{122} \mathrm{Ba},{ }^{121} \mathrm{Cs},{ }^{120} \mathrm{Xe}$ and ${ }^{118} \mathrm{Xe}$ isotopes in comparison to PACE calculation. At the initial stage it was expected that the 
modification of the statistical model input parameters could resolve the ambiguity, as the input parameters like level density, fission barrier, transmission coefficient etc are highly significant for this kind of statistical calculation. Wang et al.[3] showed that the reduction of the fission barrier could reproduce the experimentally obtained population with the statistical model calculation while compared with the statistical code ALICE (for neutron channels mainly) for the compound nuclei close to this mass region. As the isotopes populated in our experiment are very exotic neutron deficient and have large prolate deformation $\left(\beta_{2} \sim 0.25-0.30\right)$ [5], careful insertion of the input parameters like fission barrier, level density etc [4] with understanding of proper physical significance, is very important for our experimental study. The PACE calculations have been performed reducing the fission barrier (taking fission barrier scaling factor, $k_{f}=0.2,0.6$ and default $=1.0$ ) and shown in fig. 2(b). The fig. 2(b)shows that reduction of the fission barrier scale factor have not reproduced the huge experimental cross section of ${ }^{122} \mathrm{Ba},{ }^{121} \mathrm{Cs},{ }^{120} \mathrm{Xe},{ }^{118} \mathrm{Xe}$. As the evaporation residues have been populated in high excited and in high spin states, change in level density is also very important as the deformation and excitation energy, both affect the nuclear level density parameter [6]. The level density parameter constant (level density parameter, $\mathrm{a}=\mathrm{A} / \mathrm{K}$, where $\mathrm{A}=$ mass number of the compound nucleus and $\mathrm{K}$ is the level density parameter constant) has been varied to change the level density. The result thus obtained also could not reproduce the experimental result.

\section{Summary}

De-excitation of hot rotating, highly excited nuclei has been studied systematically through $\gamma$-ray spectroscopy. A number of exotic nuclei have been populated through evaporation of $\mathrm{p}, \mathrm{n}, \alpha$ etc from the excited ${ }^{124} \mathrm{Ce}$ nucleus. Population of most of the evaporation channels are in agreement with the statistical model calculation except a few multiple proton related evaporation channels. Population of ${ }^{122} \mathrm{Ba},{ }^{121} \mathrm{Cs},{ }^{120} \mathrm{Xe}$ is due to evaporation of $2 \mathrm{p}, 3 \mathrm{p}$ and $4 \mathrm{p}$ respectively from the compound nucleus. Enhanced population of these isotopes clearly point out that multiple proton evaporation channels are several times more than the statistical model prediction. It may be due to the result of enhanced proton pairing near the proton drip line [7] so that emission of multiple protons could be energetically favourable. This interesting experimental result certainly requires more detail investigation to understand the de-excitation mechanism of highly excited nuclei near proton drip line.

\subsection{Acknowledgments}

Authors are thankful to all the technical unit of the IUAC, New Delhi for their support. Author, J. Ray (CSIR Award No. 09/489(0081)/2010 EMR - 1) thanks the Council of Scientific and Industrial Research (CSIR) for their financial support. This work has been funded by the $\mathrm{XI}^{\text {th }}$ plan, SEND project (PIN:11-R\&D-SIN-5.11-0400), DAE, Govt. of India.

\section{References}

[1] J. L. Barretoet al., Physical Review C 48, pg-2881 (1993);

[2] S.Muralithar et. al, NIM A 622281287 (2010)

[3] C. Wang et al., Physical Review C 84, 014609 (2011)

[4] H. H. K Tang et al., Physical Review C 42, 1598-1622 (1990)

[5] P. Moller et al., At. Data Nucl. Tables 59, 185 (1995)

[6] S. M. Grimes et al., Physical Review C 78, 057601 (2008)

[7] G. D. Alkhazov et al., Z. Phys.A- Atoms and Nuclei 311, 245-246 (2011) 